Corresponding Author:

A M Zagrebaev

AMZagrebaev@mephi.ru

Received: 22 July 2018

Accepted: 9 September 2018

Published: 8 October 2018

Publishing services provided by Knowledge E

(c) E Yu Piskunova et al. This article is distributed under the terms of the

Attribution License, which permits unrestricted use and redistribution provided that the original author and source are credited.

Selection and Peer-review under the responsibility of the Breakthrough Directions of Scientific Research at MEPhI Conference Committee.
G OPEN ACCESS

\section{On the Refinement of the Algorithm for the Reconstruction of Missing ICMS-sensor Readings}

\section{E Yu Piskunova, R N Ramazanov, and A M Zagrebaev}

National Research Nuclear University MEPhl (Moscow Engineering Physics Institute), Department of Cybernetics, Kashirskoe shosse 31, Moscow, 115409, Russia

\section{Abstract}

Constant monitoring of the state of the core ensures safe operation of a nuclear reactor. This article examines the recovering of the energy release in the core of a nuclear reactor on the basis of the indications of height sensors. There are many cases of sensors failure. Any reduction in the number of sensor indications increases the error. The missing measurement information can be recovered by mathematical methods, and replacement of the failed sensors can be avoided. It is suggested that a set of axial and radial 'natural' functions determined by means of statistical estimates obtained from archival data be constructed. The procedure proposed makes it possible to recovery the field even with a significant loss of measurement information.

Keywords: nuclear reactor, neutron flux, loss of measuring information, sensor readings restore.

\section{Introduction}

The efficient and safety operation of a nuclear power plant (NPP) requires the continuous monitoring of the reactor core state. One important task is the control of neutrons flux density. Special sensors of in-core monitoring system (ICMS) [1] located along the core height are used to obtain information about the flux density. RBMK- reactors have assemblies of 4 self-powered neutron detectors (SPNDs), distributed uniformly over the core height. In the practice of operating nuclear reactors there may occur situations when some sensors fail. If a sensor fails, then the use of its indications is 'prohibited', e.g. assembly of SPNDs in RBMK-reactors is banned if two or more sensors fail [2]. Failure of the ICMS increases error of the neutron flux determination. The missing measurements information can be restored by mathematical methods and premature replacement of the failed sensors can be avoided. For this, it is proposed that missing sensor indications be calculated by approximation the known points. Approximation is 
suggested to be made as an expansion to the series of functions, adjusted in a certain way using the archive data [3]. The proposed technique enables to use accumulated data from an archive to restore the neutron field even after a serious loss of measurement information. There are errors in this technique, which can be partially eliminated. For this purposes, the axial and radial neutron flux distribution is used.

\section{Experiments}

Harmonic functions are used for the recovery of axial neutron flux density distribution, because they are eigenfunctions of one-dimensional nuclear reactor. The maximum number of harmonics is equal to the number of running sensors. However, these functions are not optimal for the reactor. The actual distribution of the neutron field may not be described by a superposition of these functions. In actual operation, for a neutron-physical and thermophysical feedbacks, displacement of the control rods, and others the distribution of the multiplying properties varies in time and space and is not uniform. Using these data, we obtain a matrix of the correlation coefficients. Analysis of the matrix obtained on the basis of archives of power-generating units operating in the nominal regime showed that, as a rule, the matrices are not diagonal. This means that there exists a correlation between the fitting coefficients. In other words, the neutron flux density as a random function is represented in the form of a superposition of harmonic functions with correlated random coefficients. This fact confirms the bad choice of functions for approximation.

There is the possibility to find the system of functions, which would have noncorrelated coefficients in expansion. According to the theory of random functions [4], the convergence of an expansion of a random function in the series can always be improved by an identity transformation to a canonical expansion of the random function. The procedure for transforming to the canonical expansion is reminiscent of the Gram-Schmidt procedure for orthogonalizing vectors or the method of principal components [4]. The coefficients will be expressed in terms of the correlation moments of the random quantities. We obtain an approximate canonical expansion of the random function. This approach claims that new functions called 'natural' functions are linear combinations of initial harmonic functions. Therefore, the solution of the approximation problem does not become more complex.

Xenon fluctuations constantly occur in a stationary mode along the height (see Figure 1). At the same time, deviations in the readings of the extreme SPND-sections can significantly exceed $10 \%$, especially after transient processes. This fact may be the 
reason that the error in restoring the readings in the extreme sections is higher than in the central sections, if only the values from the other SPND-sections are used for recovery.

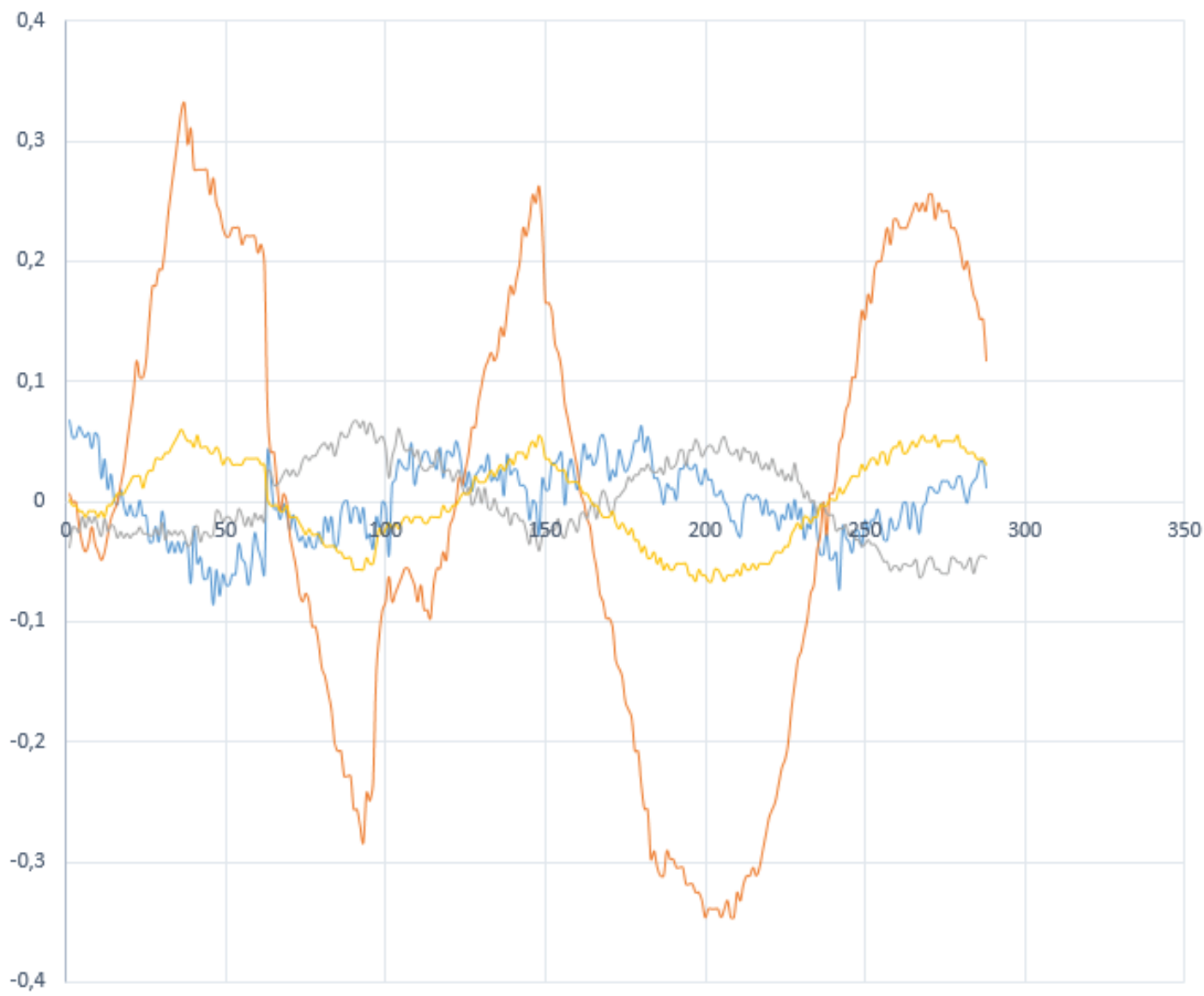

Figure 1: Relative deviation of energy release from the average over two days.

Analyze of the data archive revealed, that fluctuations occur almost inphase, it implements for a big enough field. Consequently, values of adjacent detectors, which are located in the same layer at $z$ height, will be correlated. All in all, we can use information of adjacent detectors for clarification of recovery algorithm.

Detectors of energy release are located in certain fuel channels, for each detector we can set the direction, which contain some more, along the radius of reactor core (see Figure 2).

For each such direction in four layers we can recovery the field of energy release using a set of basic functions. These functions might be eigenfunctions of cylindrical reactor, namely [6], Bessel function $J_{\alpha}$. If we set the direction along the reactor core radius for four layers, we will can recovery the field of energy release using a set of basic functions. Each set of high-altitude and radial 'natural' functions (see Figure 3 ) can be determined from the archive of the SPND readings for each channel and each 


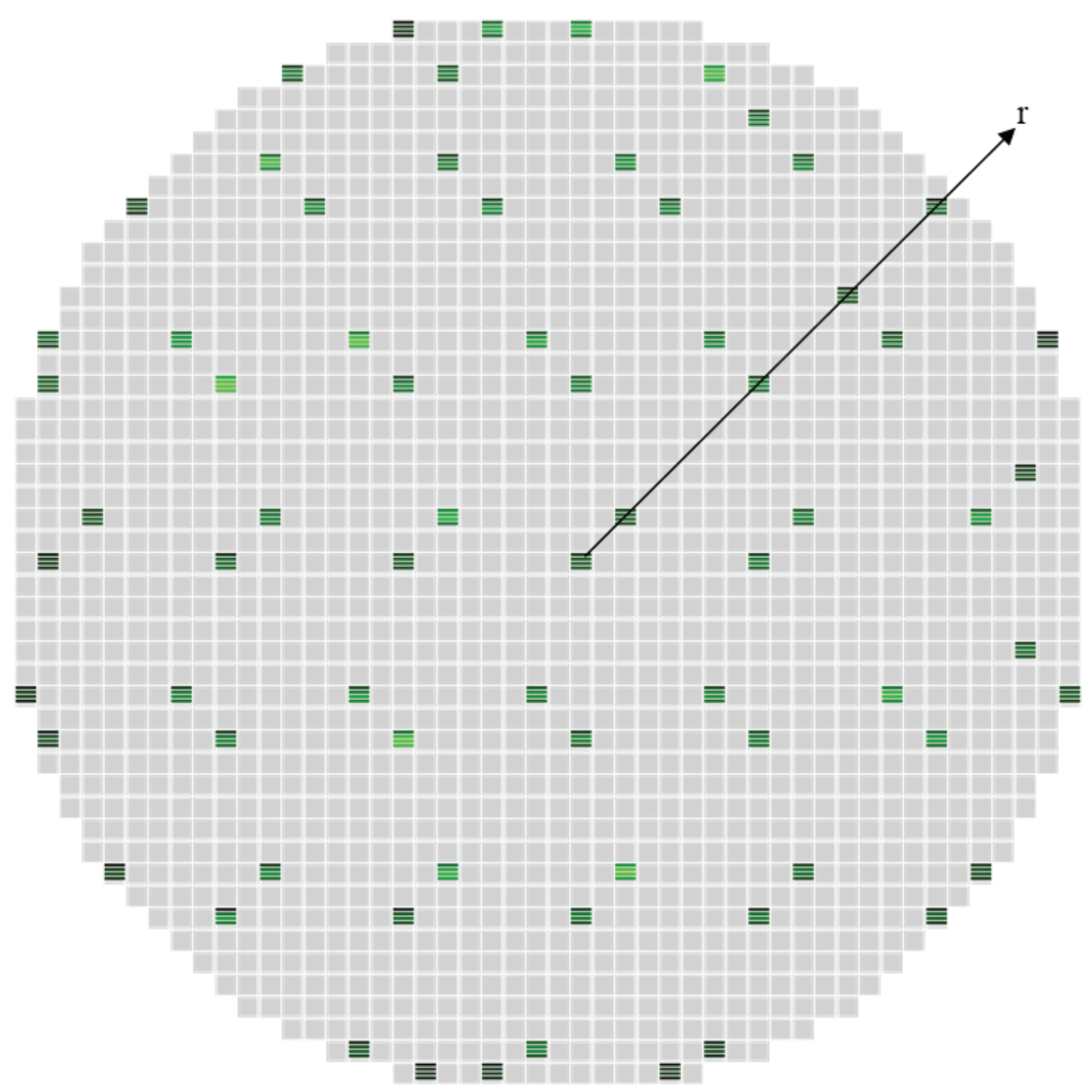

Figure 2: Location of energy release detectors on RBMK core layout.

radius. In this case, it is necessary to recalculate these functions after transient regimes or refueling near the channel with SPND.
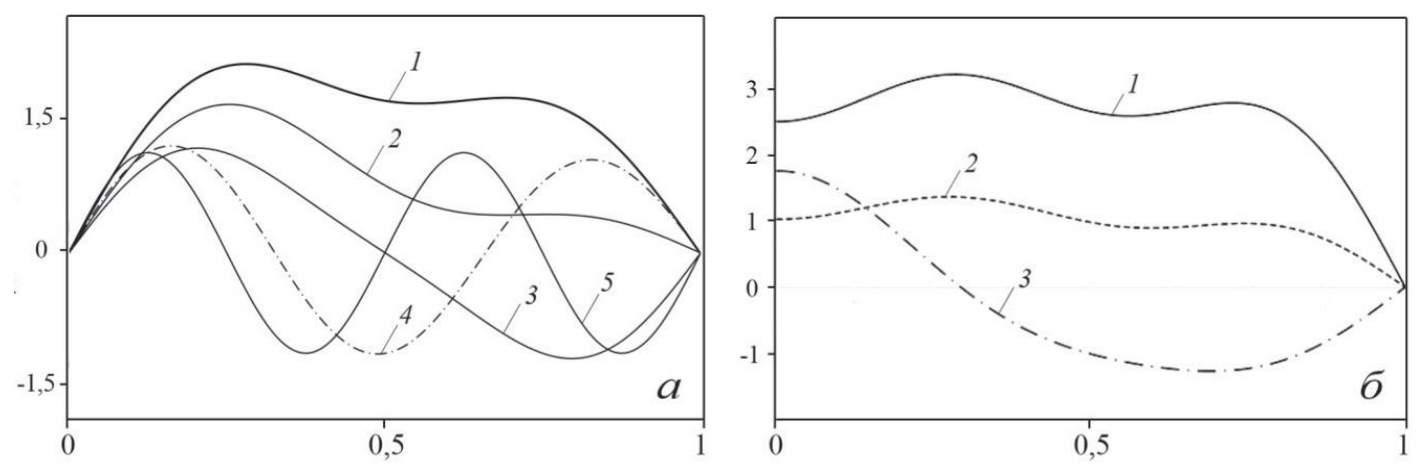

Figure 3: The 'natural' function (axial and radial approximation). 
The task of receiving uncorrelated factors comes down to representation random amplitudes in the figure of linear combinations of uncorrelated values, which have got zeroth mathematical expectations. As a result, we can receive the set of radial 'natural' functions, which are the linear combination of initial functions. Let's do research effectiveness of using different set of functions, which we seize for radial approximation. The radial 'natural' functions have advantage over elevation functions of using data of adjacent detectors, which are located in the same layer at $\mathrm{z}$ height, that provide zero deviation. Because of this, we can effectively describe the radial field using merely two 'natural' functions.

\section{Results and Discussion}

We investigate the effectiveness of using different sets of functions for axial and radial neutron flux approximating. To do this, we fictitiously 'prohibit' the sensors (i.e., do not use the corresponding indications of their sections in the calculation), and then by approximating different height and radial functions restore the lost values using the method of least squares.

The use of high-altitude 'natural' functions for restoring 'prohibited' indications in comparison with harmonic functions reduces the error by an average of 3-5 times. However, if you prohibit two sections from one edge, the error can be of the order of $10 \%$.

The recovery values using the radial 'natural' functions significantly reduce error in corporation with recovery values using cosine functions (see Figure 4). Error is less than 5 per cent for detectors, which are located in the middle part of radius, provided merely using two radial 'natural' functions. Error increase then attempted to recovery detectors, which located at the edges and at the center of reactor core.

All in all, the study showed, that using archive data from adjective detectors of energy release and preparation set of radial 'natural' functions for the sectioned radial deviation of neutron flux density, reduce recovery error. However, preferably to use elevation approximation, in case of emergency the central detectors of energy release sections, which are located at the center or at the edge of reactor core.

Therefore, the final algorithm should for each channel constantly calculate the 'natural' functions, fictitiously prohibit the readings and restore them with found functions by radius and height to select the best way to restore each section. In the case of a real section prohibition, based on previous calculations, the optimal method of recovery is selected and an appropriate set of 'natural' functions is used. Thus, the algorithm is 


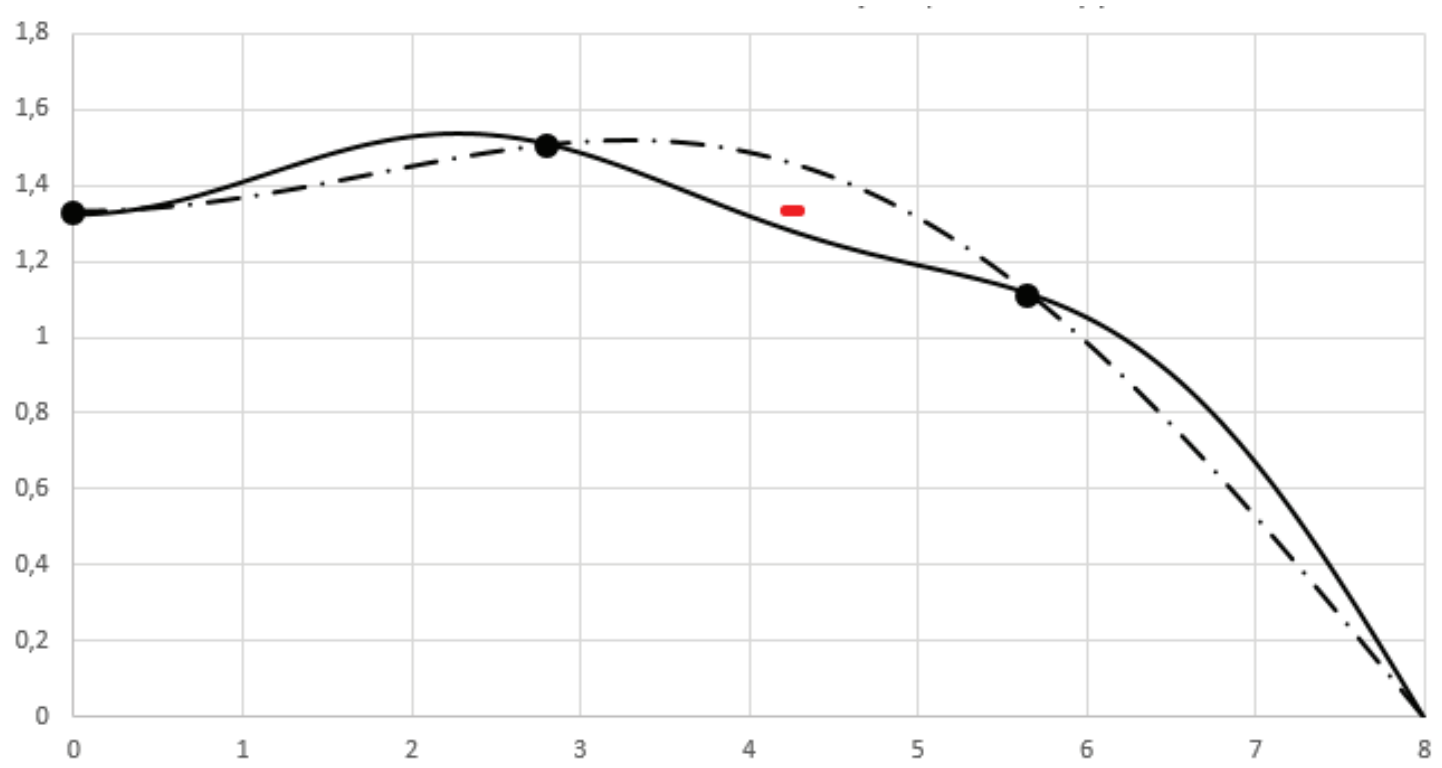

Figure 4: Two methods of recovery fictive disabled detector values (dashed line - Bessel functions, full line - 'natural' functions).

tuned during the operation of the reactor for the best recovery in the event of failure of each particular section of the SPND.

\section{Conclusions}

The use of archival information for the construction of 'natural' functions increases the accuracy of recovery of lost SPND-readings, which avoids the losses associated with the premature replacement of the fuel assembly with a faulty sensor. This effect is due to the fact that in the construction of a new basis, information is used on the possible axial and radial neutron flux distributions in the reactor operation. Moreover, on the example of solving this problem, an approach to the control of energy release is proposed, which consists in the fact that the algorithm adapts during the operation of the reactor.

\section{References}

[1] M.A. Abramov et al., Canal-type nuclear reactor RBMK (Moscow: NIKIET), 2006

[2] A.M. Zagrebayev et al., Mathematical modelling of a nuclear reactor under random perturbations of process parameters (Moscow: NRNU MEPhl), 2011

[3] A. Zagrebayev, N. Ovsyannikova, R. Ramazanov, N. Mil'to, Reconstruction of missing height-sensor readings on the basis of archival neutron field monitoring 
data (Atomic Energy 117), 2015

[4] V.S. Pugachev, Theory of random functions and its applications to control problems (Moscow: Fizmatgiz), 1960

[5] S.A. Aivazyan et al., Applied Statistics: Classification and Dimensional Reduction (Moscow: Finansy i Statistika), 1989

[6] I.Ya. Yemelyanov, A.I. Efanov, L.V. Konstantinov, Scientific and technical fundamentals of nuclear reactor management (Moscow: Energoizdat), 1981. 